\title{
Correction to: Metabolites involved in purine degradation, insulin resistance, and fatty acid oxidation are associated with prediction of Gestational diabetes in plasma
}

\author{
Lauren E. McMichael ${ }^{1} \cdot$ Hannah Heath ${ }^{1} \cdot$ Catherine M. Johnson $^{1} \cdot$ Rob Fanter $^{2,3} \cdot$ Noemi Alarcon $^{4,5}$. \\ Adilene Quintana-Diaz ${ }^{4,5} \cdot$ Kari Pilolla $^{1,5}$. Andrew Schaffner ${ }^{5,6} \cdot$ Elissa Jelalian $^{7} \cdot$ Rena R. Wing $^{7} \cdot$ Alex Brito $^{8,9}$. \\ Suzanne Phelan ${ }^{4,5} \cdot$ Michael R. La Frano ${ }^{1,3,5}$ (D)
}

Received: 10 December 2021 / Accepted: 10 December 2021 / Published online: 19 December 2021

(c) The Author(s), under exclusive licence to Springer Science+Business Media, LLC, part of Springer Nature 2021

\section{Correction to: Metabolomics https://doi.org/10.1007/s11306-021-01857-5}

In the original publication of the article, excess supplementary files were published inadvertently as ESM.

However, it has now been updated in the original article. The erratum is published just to notify this to the readers. The original article has been corrected.

Publisher's Note Springer Nature remains neutral with regard to jurisdictional claims in published maps and institutional affiliations.

Lauren E. McMichael and Hannah Heath contributed equally to warrant co-first authorship.

The original article can be found online at https://doi.org/10.1007/ s11306-021-01857-5.

Michael R. La Frano

mlafrano@calpoly.edu

1 Department of Food Science and Nutrition, California Polytechnic State University, San Luis Obispo, CA, USA

2 College of Agriculture, Food and Environmental Sciences, California Polytechnic State University, San Luis Obispo, CA, USA

3 Cal Poly Metabolomics Service Center, California Polytechnic State University, San Luis Obispo, CA, USA

4 Department of Kinesiology and Public Health, California Polytechnic State University, 1 Grand Ave, San Luis Obispo, CA 93407, USA

5 Center for Health Research, California Polytechnic State University, San Luis Obispo, CA, USA
6 Department of Statistics, California Polytechnic State University, San Luis Obispo, CA, USA

7 Department of Psychiatry and Human Behavior, Warren Alpert Medical School at Brown University, Providence, RI, USA

8 Laboratory of Pharmacokinetics and Metabolomic Analysis, Institute of Translational Medicine and Biotechnology. I.M. Sechenov First, Moscow Medical University, Moscow, Russia

9 World-Class Research Center Digital Biodesign and Personalized Healthcare, I.M. Sechenov First Moscow State Medical University, Moscow, Russia 